\title{
Introduction to the Special Edition on Social Media
}

\author{
Kenneth P. Drude ${ }^{1}[0$
}

Received: 2 April 2021 / Revised: 2 April 2021 / Accepted: 10 June 2021 / Published online: 17 June 2021

(c) The Author(s), under exclusive licence to Springer Nature Switzerland AG 2021

\begin{abstract}
This special issue of the Journal for Technology in Behavioral Science includes articles focused on some of the diverse uses and issues related to social media use by the public, clients and patients, and health care professionals. Social media broadly includes many forms of electronic communications other than Facebook and Twitter, is continuously evolving, and for many a frequent form of communicating with others. Potential benefits and risks of using social media are identified with no clear consensus on many of the issues it presents.
\end{abstract}

Keywords Social media $\cdot$ Texting $\cdot$ Facebook $\cdot$ Email $\cdot$ Twitter $\cdot$ Well-being

\section{Introduction to the Special Edition on Social Media}

Social media includes a wide range of diverse and evolving forms of communication technologies used by many people worldwide. An inclusive definition by Kaplan and Haenlein (2010) defines social media as a "group of internet-based applications that build on the ideological and technological foundations of Web 2.0, and that allows the creation and exchange of user-generated content." In this special issue of the Journal of Technology in Behavioral Science (JTiBS), "Social Media and Telebehavioral Health" we included papers that examined potential benefits and risks of using social media, factors affecting its uses, how it is being used by various populations, including health professionals, and guidelines for use of social media.

Social media has had an impact on a global scale, and the diversity of papers chosen for this special issue reflects the current state of this innovative and emerging field. The papers in this special issue include social media studies from authors from Australia, Canada, China, India, Nigeria, United States, and United Kingdom. We aimed to include papers representing the diversity of research in social media, including uses in healthcare settings, health impacts, and the use of social media in combination with other technologies, such as email and texting.

Kenneth P. Drude

Kdrude@yahoo.com

1 Dayton, OH, USA
In this special issue we aim to inform behavioral health practitioners, educators, trainers, the general public, and professional and health organizations regarding the increasing uses in healthcare settings, the potential impact social media has on many aspects of society, and provide guidance regarding best practices for use in healthcare settings.

\section{Professional Uses of Social Media}

Social media communications use by healthcare professionals continues to present ethical challenges, particularly for regulatory boards that are charged with enforcing licensure laws and rules, even though a number of national health professional organizations have developed and adopted social media guidelines to provide guidance in this regard. The paper by Drude and Messer-Engel (2020) described the development and adoption of social media guidelines by the Association of State and Provincial Psychology Boards, the national organization for the 65 separate psychology licensing boards in Canada and the United States and its territories. A major goal was to provide a shared perspective for all jurisdictions in both countries regarding the interpretation and application of ethical standards in the use of social media by psychologists in both countries.

Two papers focus on examples of how social media was used in providing or promoting mental health and or substance abuse treatment to consumers. Guenzel et al., 2021 found that providing a social media app (Sober Grid) to adults in an outpatient addiction recovery program after they completed an inpatient addiction program was a valuable complement 
to their treatment in reducing relapse. The authors emphasized that different participants used the app in different ways for peer support including chatting with other program participants for support, providing support to others and to "chat with others to build a positive social network." The paper by Hong et al., 2021 demonstrated an example of how Facebook and Twitter were successfully used to decrease the stigma of military service members seeking mental health services and to promote the use of those services. This paper described a social media campaign developed by the United States Department of Defense and the analysis of the impact of Facebook and Twitter posts shared. Based upon the content analysis of the messages, they determined the quality of the messages that had the most impact on behavior. Findings emphasized the importance of knowing the culture of the target audience, which allowed more targeted messaging. As a result of these changes Facebook and Twitter messages, shares, and retweet significantly increased and the number of individuals seeking help increased.

The study by Ukoha and Stanieri, 2021 included a survey of Australian hospitals and medical clinics to learn how health organizations use social media communications. The authors found a wide diversity of uses including those that were patient focused (education, obtaining feedback, patient collaboration), professional focused (engaging in professional networking, education, conducting research), and general public health focused (engaging the public, promoting public health, crowdsourcing).

\section{Social Media Use and Personal Impact or Relationships}

Relating social media use to characteristics of the users such as well-being and mental health and negative health outcomes is a common theme in social media studies. Many papers in this special issue examined this, and a diversity of results was described.

The mental health or well-being of social media users and their use of social media are studied in papers by Al-Saffaf, 2021; Sindermann et al., 2021 and Keyte et al., 2021. Al-Saggaf, 2021 and colleagues investigated the relationship of phubbing (or ignoring others present and giving attention to a smart phone), feeling bored, and fear of missing out (FOMO). They found that the fear of missing out acted as an "excuse to phub in order to avoid or alleviate the negative feeling of state boredom." Another finding was that as the age of users increased, phubbing decreased. The study by Sindermann et al., 2021 looking at problematic uses of the social app, WeChat, by college students and faculty, examined a neuroticism self-rating of users and the mediating role of FOMO. This study positively related uses of WeChat that negatively impacted the user's daily lives and well-being to neuroticism ratings that were also significantly related to FOMO. Thirty-six percent of the study participants obtained use ratings that the author posed "...might indicate an actual pathological overuse of WeChat."

The issue of "addiction," or excessive use of social media, was an underlying theme in several studies. A strong positive relationship between motor vehicle drivers with "mobile phone addiction proneness" and road accidents by Adeyemi, 2021 is an example. Adeyemi, 2021 related self-reported road accidents in Nigeria to the drivers' ratings on a Smartphone Addiction Survey - Short Version and found that drivers prone to mobile phone addition were $2 \frac{1}{2}$ times more likely to have had an accident linked directly or indirectly to mobile phone use while driving.

Arora et al., 2021 studied the impact of the COVID-19 pandemic lockdown on Twitter users in India by analyzing the emotional content of tweets and the frequency of tweets by users. Tweets were analyzed for major emotional states, including happiness, sadness, anger, fear, disgust, and surprise. A significant number of users (40.1\%) expressed happiness during the study and those with the most frequent tweets tended to express negative emotions (anger, disgust, or sadness) in their tweets.

The content analysis of Twitter tweets originating from Puerto Rico and the continental United States following Hurricane Maria by Rodriguez-Guzman et al., 2021 found that users tweeting from Puerto Rico had more positive emotional content than those from elsewhere. Interestingly, tweets from outside of Puerto Rico more frequently referenced death than those originating from Puerto Rico. The authors concluded that Twitter users from Puerto Rico were less focused on the past or future.

Instagram use by young adults and its relationship to their well-being was explored by Keyte et al., 2021. They found a strong positive correlation between the frequency of Instagram usage and well-being as measured by self-ratings of depression, anxiety, and stress, and a negative relationship between self-compassion and time spent using Instagram and poorer well-being. The authors asserted that “...Instagram intensity appeared to influence psychological well-being, with participants who spent more time on Instagram reporting poorer psychological well-being...".

A qualitative study by Shensa et al., 2021 compared the negative and positive social media experiences of a sample of young adults in the United States and examined the potential impact on their mental health as measured by the selfreported depressive symptoms as measured by the PHQ-9 of the study participants. One of several significant findings was that participants that reported severe depressive symptoms (compared to those with no symptoms) indicated spending greater amounts of time using social media and using a greater number of different platforms. Both positive and negative social media themes were identified by 
participants with those with more depressive symptoms more often perceiving negative effects than those with no depressive symptoms.

An opinion paper by Blake and Sauermilch, 2021 is a cautionary commentary about labeling LBGQT + adolescents as having an Internet gaming disorder. They emphasized that parents and health professionals ought to consider the potential benefits that online gaming may provide to some LBGQT + adolescents and avoid assuming that it is addictive and a "problem." They posited that "[n]eglecting the psychosocial benefits of gaming for such populations during a critical phase of development would not only further contribute to the marginalization of sexual and gender minority populations broadly, but would also undercut our own understanding of the mental health implications of gaming at large."

The survey of college students in Hong Kong, the United States, and the United Kingdom by Przybylski et al. 2021 that looked for a relationship between briefly abstaining from using different social media and social relationships, positive and negative affect, and satisfaction did not find a positive relationship. Study participants were asked to not use any of ten different social media platforms for a day at a time. The abstinence compliance rate was relatively low (49.5\%) and those that did comply reported lower rather than higher in-person, telephone, or email interactions. The authors concluded that their findings indicated that taking a brief abstinence from social media did not have a measurable positive effect on well-being.

The Technology Acceptance Model was used by Kong et al., 2021 in their study to examine the relationship of attitudes about technology and its uses on the use of social media. Their study of college students showed that the perceived usefulness and ease of use of mobile social media were positively influenced their intended and actual use of social media.

We feel that the breadth and depth of the topics covered in the current special issue provide a good overview of the current state of field regarding the health impacts and uses of social media. While this is an emerging area of research, the impact of social media has been remarkable, and the potential for use is yet to be seen.

Acknowledgements The author acknowledges and much appreciates the assistance of Christina Armstrong, Myo Thwin T. Myint, and John Nashlund in developing this special issue.

\section{Declarations}

Conflicts of Interest The author declares no competing interests.

\section{References}

Adeyemi, O. (2021). The association of mobile phone addiction proneness and self-reported road accident in Oyo State, Nigeria. Journal of Technology in Behavioral Science. Brief Report Published February 2021.

Al-Saggaf, Y. (2021). Phubbing, fear of missing out and boredom. Journal of Technology in Behavioral Science, 6(2), 352-357. Original Paper Published January 2021.

Arora, A., Chakraborty, P., Bhatia, M. P. S., \& Mittal, P. (2021). Role of emotion in excessive use of Twitter during COVID-19 imposed lockdown in India. Journal of Technology in Behavioral Science, 6(2), 370 - 377. Original Paper Published October 2020.

Blake, E., \& Sauermilch, D. (2021). Reconsidering internet gaming disorder during the COVID-19 pandemic. Journal of Technology in Behavioral Science, 6(2), 348-351. Original Paper Published January 2021.

Drude, K. \& Messer-Engel, K. (2020). The development of social media guidelines for psychologists and for regulatory use. Journal of Technology in Behavioral Science, 6(2), 388-396. Original Paper Published November 2020.

Guenzel, N., McChargue, D., \& Dai, H. (2021). Testing the use of a social networking app for American Indians recovering from addiction. Journal of Technology in Behavioral Science. Brief Report Published February 2021.

Hong, E., Slay, P., Hampton, S., Critchfield, D., Wenzlaff, T., Castille, K., Polizzi, N., and Hoyt, T. (2021). Illustrating best practices in optimizing social media strategy for a campaign targeting military mental health stigma. Journal of Technology in Behavioral Science, 6(2), 427-435. Original Paper Published January 2021.

Kaplan, A. M., \& Haenlein, M. (2010). Users of the world, unite! The challenges and opportunities of social media. Business Horizons, 53(1), 59-68. https://doi.org/10.1016/j.bushor.2009.09.003

Keyte, R., Mullis, L., Hussain, M., Egan, H., Cook, A., and Mantzios, M. (2021). Self-compassion and Instagram use Is explained by the relation to anxiety, depression, and stress. Journal of Technology in Behavioral Science, 6(2), 436-441. Original Paper Published December 2020.

Kong, F., Xia, Y., Wang, M., \& Sun, X. (2021). Technology acceptance model of mobile social media among Chinese college students. Journal of Technology in Behavioral Science, 6(2), 365-369. Original Paper Published September 2020

Przybylski, A. K., Nguyen, T., Law, W., \& Weinstein, N. (2021). Does taking a short break from social media have a positive effect on wellbeing? Evidence from three preregistered field experiments. Journal of Technology in Behavioral Science. Published February 2021.

Rodríguez-Guzmán, V., Garcia-Ramirez, G., Bogen, K., Orchowski, L. and Nugent, N. (2021). PuertoRicoSeLevanta: A closer look at the language used on the first-year anniversary of hurricane Maria. Journal of Technology in Behavioral Science, 6(2), 358364. Original Paper Published September 2020.

Shensa, A., Sidani, J., \& Burke, J. (2021). Positive and negative social media experiences among young adults with and without depressive symptoms. Journal of Technology in Behavioral Science, 6(2), 378-387. Original Paper Published October 2020.

Sindermann, C. , Yang, H., Liu, T., Elhai, J., and Montag, C. (2021). WeChat-Its Problematic Use and Relations with the Big Five Personality Traits and Fear of Missing Out. Journal of Technology in Behavioral Science, 6(2), 397-405. Original Paper Published January 2021. 
Ukoha, C., \& Stranieri, A. (2021). On the Value of Social Media in Health Care. Journal of Technology in Behavioral Science, 6(2), 419-426. Original Paper Published January 2021.
Publisher's Note Springer Nature remains neutral with regard to jurisdictional claims in published maps and institutional affiliations. 